\title{
IL-5 links adaptive and natural immunity specific for epitopes of oxidized LDL and protects from atherosclerosis
}

\author{
Christoph J. Binder, ${ }^{1}$ Karsten Hartvigsen, ${ }^{1}$ Mi-Kyung Chang, ${ }^{1}$ Marina Miller, ${ }^{2}$ David Broide, ${ }^{2}$ \\ Wulf Palinski, ${ }^{1}$ Linda K. Curtiss, ${ }^{3}$ Maripat Corr, ${ }^{2}$ and Joseph L. Witztum ${ }^{1}$
}

${ }^{1}$ Division of Endocrinology \& Metabolism, 2Division of Rheumatology, Allergy \& Immunology, Department of Medicine, University of California, San Diego (UCSD), La Jolla, California, USA. ${ }^{3}$ Department of Immunology, The Scripps Research Institute, La Jolla, California, USA.

\begin{abstract}
During atherogenesis, LDL is oxidized, generating various oxidation-specific neoepitopes, such as malondialdehyde-modified (MDA-modified) LDL (MDA-LDL) or the phosphorylcholine (PC) headgroup of oxidized phospholipids (OxPLs). These epitopes are recognized by both adaptive $T$ cell-dependent (TD) and innate $T$ cell-independent type 2 (TI-2) immune responses. We previously showed that immunization of mice with MDA-LDL induces a TD response and atheroprotection. In addition, a PC-based immunization strategy that leads to a TI-2 expansion of innate B-1 cells and secretion of T15/EO6 clonotype natural IgM antibodies, which bind the PC of OxPLs within oxidized LDL (OxLDL), also reduces atherogenesis. T15/EO6 antibodies inhibit OxLDL uptake by macrophages. We now report that immunization with MDA-LDL, which does not contain OxPL, unexpectedly led to the expansion of T15/EO6 antibodies. MDA-LDL immunization caused a preferential expansion of MDA-LDL-specific Th2 cells that prominently secreted IL-5. In turn, IL-5 provided noncognate stimulation to innate B-1 cells, leading to increased secretion of T15/EO6 IgM. Using a bone marrow transplant model, we also demonstrated that IL-5 deficiency led to decreased titers of T15/EO6 and accelerated atherosclerosis. Thus, IL-5 links adaptive and natural immunity specific to epitopes of OxLDL and protects from atherosclerosis, in part by stimulating the expansion of atheroprotective natural IgM specific for OxLDL.
\end{abstract}

\section{Introduction}

Atherosclerosis is a chronic inflammatory disease $(1,2)$ whose pathogenesis involves disturbed lipoprotein metabolism, the formation of proinflammatory lipid peroxidation products, and the host's immune responses $(3,4)$. Oxidized LDL (OxLDL) is present in atherosclerotic lesions and contains a wide variety of lipid peroxidation products, which in turn can form neo-self determinants recognized by specific innate and adaptive immune responses $(3,4)$.

Typically, peroxidation of the abundant phospholipid phosphatidylcholine is initiated at the oxidation-prone $s n-2$ polyunsaturated fatty acid. Its subsequent decomposition generates a wide spectrum of reactive molecular species, such as malondialdehyde (MDA) and the residual core-aldehydes of the oxidized phospholipids (OxPLs) that contain the phosphorylcholine (PC)-headgroup, such as 1-palmitoyl-2-(5-oxovaleroyl)-sn-glycero3 -phosphorylcholine (POVPC). These reactive aldehydes can modify autologous molecules, including the LDL particle, generating multiple immunogenic oxidation-specific epitopes.

Specific humoral responses to different oxidation-specific epitopes of OxLDL have been described (5), but their role in health and disease as well as their immunobiological orchestration are

Nonstandard abbreviations used: alkaline phosphatase (AP); bone marrow transplantation (BMT); fast-performance liquid chromatography (FPLC); immune complex (IC); LDL receptor (LDLR); malondialdehyde (MDA); MDA-modified LDL (MDA-LDL); oxidized LDL (OxLDL); oxidized phospholipid (OxPL); 1-palmitoyl-2(5-oxovaleroyl)-sn-glycero-3-phosphorylcholine (POVPC); phosphorylcholine (PC); relative light units (RLU); T cell-dependent (TD); T cell-independent (TI); T cellindependent type 2 (TI-2).

Conflict of interest: The authors have declared that no conflict of interest exists.

Citation for this article: J. Clin. Invest. 114:427-437 (2004)

doi:10.1172/JCI200420479. largely unknown. IgM and IgG autoantibodies to OxLDL develop in humans and in animal models of atherosclerosis, and their titers often correlate with the extent of disease (5). We have extensively characterized IgM monoclonal anti-OxLDL autoantibodies that were derived from the spleens of atherosclerotic ApoE-deficient mice, as these autoantibodies partially reflect the disease-associated immune responses (6-9). In vitro, most of these IgMs bound to OxLDL, which is made by copper-catalyzed oxidation of LDL and therefore contains many different oxidation products, including oxidized phospholipids, such as POVPC. Genetic analysis indicated that EO6, the prototypic IgM anti-OxLDL antibody, was a natural antibody secreted by innate B-1 cells in a $T$ cell-independent (TI) manner and possessed the germline-encoded T15 clonotype (8). T15 antibodies have previously been shown to recognize the PC moiety (10), which is an immunodominant component of the cell wall of many microbes, such as Streptococcus pneumoniae (11). Our previous studies show that T15/EO6 also recognizes the PC moiety of oxidized phospholipids, as present on OxLDL and apoptotic cells, but does not recognize native PC-containing unoxidized phospholipids, as found on native LDL or viable cells (8). Thus, oxidation of phosphatidylcholine "exposes" the PC moiety, making it an epitope for T15/EO6 or a ligand for scavenger receptors, such as CD36 (3). Indeed, the IgM EO6 is able to block the uptake of OxLDL by macrophages in vitro, preventing foam cell formation $(7,8)$. Furthermore, immunization of LDL receptordeficient ( $\left.L D L R^{-/-}\right)$mice with $S$. pneumoniae, which results in the near monoclonal expansion of IgM T15/EO6 antibodies, exerts a protective effect on lesion formation (12).

IgG antibodies to OxLDL epitopes could potentially be detrimental by promoting macrophage foam-cell formation via Fcy receptors. However, we $(13,14)$ and others $(15,16)$ have shown that active 
immunization of hypercholesterolemic rabbit and murine models of atherosclerosis with an oxidation-specific epitope - homologous MDA-modified LDL (MDA-LDL) - induces robust T cell-dependent (TD) IgG titers and reduces the progression of atherogenesis, suggesting that components of the adaptive immune response to these antigens can also be protective. MDA-LDL is prepared by chemical derivatization of native LDL, and though it contains many MDA epitopes, the LDL itself is not oxidized and therefore does not contain other oxidation-specific epitopes, such as oxidized phospholipids recognized by EO6 $(6,7)$.

There is strong evidence that adaptive immunity is involved in the natural course of atherogenesis $(3,17)$, and substantial evidence supports a disease-promoting role for Th1 responses and Th1 cytokines in particular (17). Specifically, adaptive responses to OxLDL have been shown to increase during disease progression, but their exact role in atherogenesis is uncertain $(18,19)$. Notably, in cholesterol-fed apoE $E^{-/-}$mice, advanced stages of atherosclerosis are associated with increased accumulation of OxLDL (20), and in this setting there is an immune deviation of OxLDL-specific Th1 responses toward Th2 $(21,22)$. The Th2 cytokine IL- 4 has been reported to have either pro- or anti-atherogenic effects (23-25), whereas IL-10 secretion by T cells decreases atherogenesis $(26,27)$. Thus, it is not known what effect a general Th2 immune deviation may have on disease progression.

Immunization with MDA-LDL induces a specific TD response $(14,16)$. In the course of studies attempting to define the mechanisms of the protective effect of MDA-LDL immunization, we found a marked Th2 bias of the induced MDA-specific TD responses that were characterized by prominent secretion of the Th2 cytokine IL-5. Surprisingly, there was a parallel induction of TI anti-PC T15/EO6 antibodies, which do not recognize MDA modifications. We further established that IL-5 could stimulate these natural TI humoral responses to oxidized phospholipid epitopes in vivo and in vitro in a noncognate manner, and we demonstrated the importance of this by showing that IL-5 deficiency accelerated atherosclerosis. These data support a paradigm in which an antigen-driven specific Th2 response not only leads to classic TD responses, but in turn enhances innate humoral responses to other oxidation-specific epitopes of OxLDL, which in aggregate provide protection from atherosclerosis.

\section{Results}

Immunization with MDA-LDL induces a specific Th2-biased response. We first immunized normocholesterolemic C57BL/6 mice with homologous MDA-LDL in Freund's adjuvant and examined the antigen-specific proliferation in splenic cultures. Splenocytes from immunized but not naive mice exhibited dose-dependent proliferation in response to MDA-LDL, but not to native LDL (Figure 1A). We next quantified titers of TD antibody isotypes to MDA-LDL in plasma. Measurements from three independent studies revealed more than an eight-fold greater induction of MDA-LDL-specific IgG1 titers over IgG2a titers $(P<0.01)$, demonstrating a strong Th2 bias of the induced response (Figure $1 \mathrm{~B}$ ), which occurred despite the use of CFA in the C57BL/ 6 genetic background that typically results in Th1 responses (28). Studies in which $M H C$ class $\mathrm{II}^{-/-}$and T-cell receptor $\beta^{-/-}$mice were injected with MDA-LDL indicated that the IgG responses to MDA-LDL were dependent on MHC II class antigen presenting cells and $\alpha \beta \mathrm{T}$-cell receptor-expressing $\mathrm{T}$ cells (data not shown). In parallel studies, we also immunized C57BL/6 mice with MDA-LDL, this time without adjuvant. Although fewer than half of these animals developed an antigen-specific titer, again $\operatorname{IgG} 1$ was the dominant isotype in the responding mice, and even at plasma dilutions as low as 1:50, no IgG2a binding was detected (data not shown).

To demonstrate the dominant Th2 responses, we quantified the relative frequencies of MDA-LDL-specific Th1 and Th2 cells in splenocyte cultures by enzyme-linked immunospot (ELISpot) assay using IFN- $\gamma$ and IL-5 as surrogate Th 1 and Th 2 cytokines, respectively. After overnight incubation with MDA-LDL, an
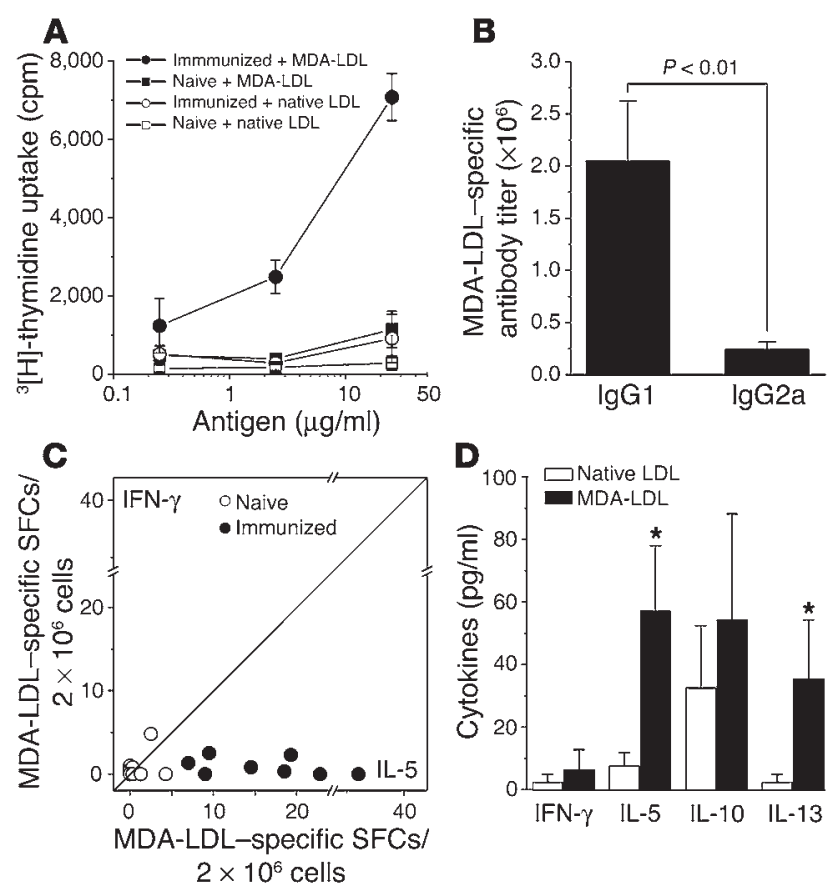

\section{Figure 1}

Immunization with MDA-LDL induces a specific Th2 response. C57BL/6 mice were immunized with homologous MDA-LDL in Freund's adjuvant or remained naive. One week after the third injection, cellular and humoral immune responses were assessed. Three independent immunization studies were performed. (A) Splenocyte proliferation assay. Splenocytes of immunized $(n=6)$ or naive $(n=6)$ mice were cultured with titrated amounts of murine native LDL (open circles, immunized; open squares, naive) or murine MDA-LDL (filled circles, immunized; filled squares, naive), and antigen-specific proliferation was measured by ${ }^{3}[\mathrm{H}]$-thymidine uptake. (B) Increased IgG1 titers in plasma of immunized mice. Data represent titers from all mice studied $(n=14 ; P<0.01$, Student's paired $t$ test). (C) Increased frequency of MDA-LDL-specific IL-5 secreting cells in spleens of immunized mice as assessed by ELISpot assay. Splenocytes of immunized ( $n=8$; filled circles) and naive ( $n=8$; open circles) mice were incubated overnight with and without murine MDA-LDL, and the frequencies of MDA-LDL-specific IFN- $\gamma$ or IL- 5 spot-forming cells (SFCs) were assessed. Shown are the mean SFCs per $2 \times 10^{6}$ cells for IFN- $\gamma$ and IL- 5 of individual mice from two experiments $(P<0.01$, Student's paired $t$ test; IFN- $\gamma$ vs. IL-5 SFC of immunized mice). (D) MDA-LDL-specific Th2 cytokine secretion in cultures of splenocytes from four mice incubated for 72 hours with 25 $\mu \mathrm{g} / \mathrm{ml}$ murine native LDL (white bars) or murine MDA-LDL (black bars). Control cultures stimulated with anti-CD3 and anti-CD28 produced $11.7 \pm 2.1 \mathrm{ng} / \mathrm{ml} \mathrm{IFN}-\gamma, 156 \pm 39 \mathrm{pg} / \mathrm{ml} \mathrm{IL}-5,470 \pm 43 \mathrm{pg} / \mathrm{ml} \mathrm{IL}-10$, and $1.2 \pm 0.4 \mathrm{ng} / \mathrm{ml} \mathrm{IL-13.}{ }^{*} P<0.05$; MANOVA followed by NewmanKeuls test, comparing the stimulation with increasing amounts $(0.25$ [not shown], 2.5 [not shown], and $25 \mu \mathrm{g} / \mathrm{ml}$ ) of native and MDA-LDL. All values shown in Figure 1 are mean \pm SEM. 


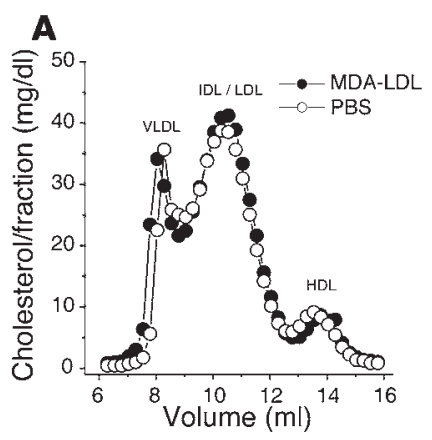

$\mathbf{B}$
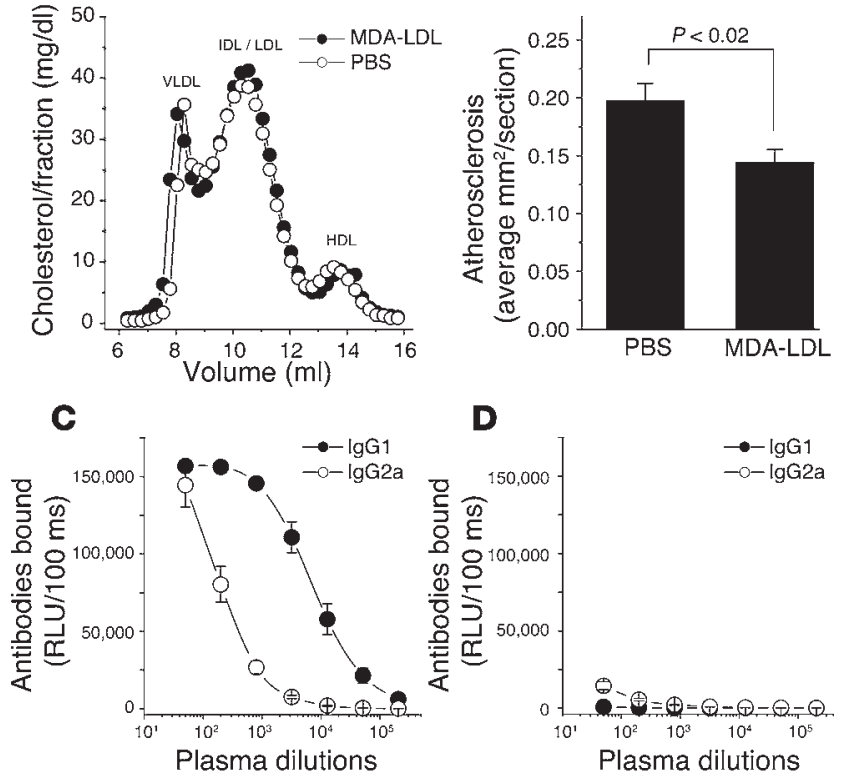

E
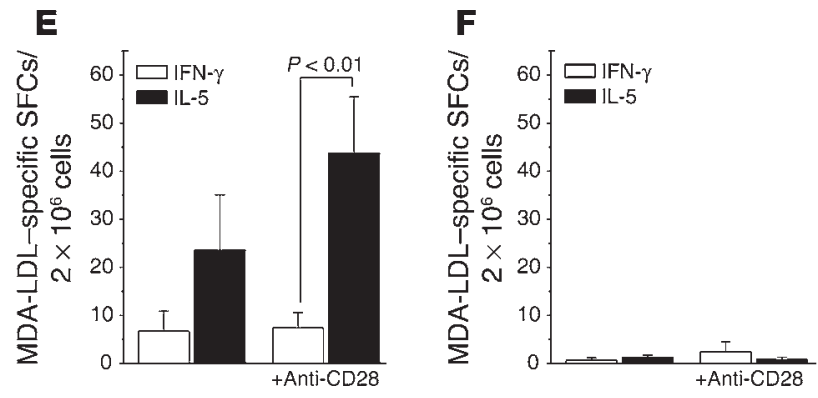

increased and significantly higher frequency of IL-5 secreting cells specific for MDA-LDL could be detected $(P<0.01)$ compared with an only minimal induction of specific IFN- $\gamma$-secreting cells in the spleens of the same mice (Figure 1C). Splenocytes from naive mice showed no response.

In addition, measurement of cytokines present in the supernatants of splenocytes from immunized mice cultured with MDA-LDL revealed an MDA-LDL-specific secretion of Th2 cytokines IL-5, IL-10, and IL-13, but not of the Th1 cytokine IFN- $\gamma$ (Figure 1D). Supernatants from splenocytes cultured with native LDL did not secrete appreciable levels of these cytokines, with the exception of IL-10, which was not consistent in every experiment (Figure 1D). Splenocytes from naive control mice, cultured with either MDALDL or native LDL, did not exhibit specific cytokine secretion (data not shown).

Th2 responses persist in MDA- $L D L-i m m u n i z e d$ mice with atherosclerosis. To determine whether such a Th2 bias in response to MDA-LDL immunization would also occur in mice with developing atherosclerosis, which has been associated with increased Th1 responses (17), we immunized cholesterol-fed $L D L R^{-/}$mice with MDA-LDL. Mice were initially immunized twice with either $\operatorname{MDA}-\operatorname{LDL}(n=10)$ or PBS $(n=11)$ emulsified in Freund's adjuvant and were then fed a high-cholesterol diet for 13 weeks, during which time three additional boosts were given. At the end of this intervention, mice in the MDA-LDL and PBS groups gained equal weight (27.5 $\pm 0.9 \mathrm{~g}$ vs. $27.5 \pm 0.7 \mathrm{~g})$ and had similar total plasma cholesterol $(1,119 \pm 17$ $\mathrm{mg} / \mathrm{dl}$ vs. $1,114 \pm 34 \mathrm{mg} / \mathrm{dl})$ and triglyceride levels $(224 \pm 12 \mathrm{mg} / \mathrm{dl}$

\section{Figure 2}

Decreased atherosclerosis and dominant Th2 response in immunized $L D L R^{-/-}$mice. Mice were immunized with homologous MDA-LDL $(n=10)$ or PBS $(n=11)$ in Freund's adjuvant, and then fed a high-cholesterol diet for 13 weeks, during which they received further booster immunizations. (A) Lipoprotein profiles at time of death in pooled plasma of all mice immunized with MDA-LDL (filled circles) or PBS (open circles) as determined by FPLC. (B) Decreased atherosclerotic lesion size in cross-sections through the aortic origin in mice immunized with MDA-LDL. Values are mean \pm SEM in $\mathrm{mm}^{2} /$ section. (C and D) Plasma IgG1 (filled circles) and IgG2a (open circles) dilution curves of binding to MDA-LDL of mice immunized with (C) MDA-LDL or (D) PBS. Values are the mean \pm SEM of all final plasma samples for each group measured in duplicate. (E and F) ELISpot assay of frequencies of MDA-LDL-specific cytokine-secreting cells in the spleens of mice immunized with (E) MDA-LDL or (F) PBS. Splenocytes were incubated overnight in the absence or presence of murine MDA-LDL with and without anti-CD28, and the frequencies of MDA-LDL-specific IFN- $\gamma$ (white bars) or IL-5 (black bars) SFCs were assessed. Bars represent the mean SFCs \pm SEM of $2 \times 10^{6}$ cells of all mice for each group. $P<0.01$, Student's paired $t$ test.

vs. $251 \pm 28 \mathrm{mg} / \mathrm{dl}$ ). Both groups of mice had identical lipoprotein profiles, as measured by fast-performance liquid chromatography (FPLC; Figure 2A). Despite the marked hypercholesterolemia, consistent with earlier studies $(13,14)$, MDA-LDL immunization led to significantly reduced $(P<0.02)$ atherosclerosis in the aortic origin (Figure $2 \mathrm{~B}$ ), the site where atherosclerotic lesions first develop in mice. However, the percentage of surface area of the arch covered by lesions was not significantly different at this site between the two groups $(10.3 \pm 1.0 \%$ vs. $10.9 \pm 1.0 \%)$.

Similarly to the normocholesterolemic C57BL/6 mice (Figure 1B), immunized cholesterol-fed $L D L R^{-/-}$mice developed IgG1 titers to MDA-LDL that were more than 2 logs higher than the IgG2a titers (Figure $2 \mathrm{C}$ ). In contrast, $L D L R^{-/-}$mice injected with PBS in Freund's adjuvant developed minimal IgG2a titers to MDA$\mathrm{LDL}$ as a result of the atherogenic diet, but no specific IgG1 titers were induced (Figure 2D). This Th2 bias was further confirmed by the study of MDA-LDL-specific T cells in the spleens of these mice. ELISpot analysis for cells secreting IFN- $\gamma$ or IL-5 revealed an increased frequency of IL-5-secreting cells specific for MDA-LDL in the spleens of MDA-LDL-immunized mice (Figure 2E), but not in the spleens of $L D L R^{-/-}$mice injected with PBS in Freund's adjuvant (Figure 2F). This pattern of response was further enhanced by the addition of anti-CD28 for in vitro costimulation of these MDA-LDL-specific T cells previously committed in vivo (Figure $2 \mathrm{E})$. Splenocytes from $L D L R^{-/-}$control mice displayed a low frequency of MDA-LDL-specific IFN- $\gamma$-secreting cells, even when cultures were costimulated with anti-CD28 (Figure 2F), consistent with the moderate IgG2a titers to MDA-LDL (Figure 2D). Thus, even during the development of atherosclerosis, which is accompanied by many proinflammatory stimuli associated with Th1 responses (17), MDA-LDL-specific Th2 responses remained dominant in immunized mice.

$I L-5$ is prominently secreted by $M D A-L D L-$ specific $T$ cells. To further define the Th2 response in the MDA-LDL-immunized $L D L R^{-/-}$ mice, we quantified the cytokines secreted by splenocytes from immunized mice cultured with MDA-LDL or native LDL, in the presence of anti-CD28 to provide sufficient costimulation. To identify the dominant cytokine secreted by MDA-specific $\mathrm{T}$ cells, we stimulated parallel cultures with anti-CD3 and anti- 
CD28 (anti-CD3/anti-CD28) to achieve maximal nonspecific cytokine release and compared the results with the cytokine release obtained after stimulation with MDA-LDL (Figure 3, left panels). Cytokine production in response to anti-CD3/antiCD28 did not differ between the two groups (data not shown). In cultures with native LDL, no substantial cytokine secretion was observed. Splenocyte cultures from $L D L R^{-/-}$mice that received PBS in Freund's adjuvant exhibited no specific cytokine secretion (Figure 3, left panels). Strikingly, MDA-LDL-specific secretion of IL- 5 by T cells of MDA-immunized mice was greater than $75 \%$ of the amount achieved with maximal nonspecific stimulation in parallel cultures. By comparison, MDA-LDL-specific secretion of other Th2 cytokines (IL-4, IL-10, IL-13) was only $20-40 \%$ of that induced by anti-CD3/anti-CD28. In contrast, MDA-LDL-specific IFN- $\gamma$ secretion was negligible. This pattern of response was also seen in cultures where dose-dependent cytokine secretion was assessed in response to stimulation with MDA-LDL alone (i.e., without additional costimulation; Figure 3, right panels).

Plasma levels of IL-5 are increased by MDA-LDL immunization. To assess whether IL-5 was also increased in vivo, we measured cytokine levels in the plasma of all mice at the end of the intervention study. MDA-LDL-immunized mice had significantly higher plasma levels of IL-5 when compared to PBS-immunized mice (Figure 4A). Plasma IFN- $\gamma$ levels were much lower and close to the quantification limit $(50 \mathrm{pg} / \mathrm{ml})$, with no measured differences between the two groups (data not shown).

MDA-LDL immunization increases T15/EO6 natural IgM antibodies to $O x L D L$. Consistent with a previous study (14), the MDA-LDL immunization led to significant increases in IgM titers to MDALDL (data not shown), but surprisingly, we also found increased titers of IgM to OxLDL, and increased titers of IgM specific to the PC of OxPLs (data not shown). As noted, such epitopes are not present in the model MDA-LDL used as an immunogen $(6,7)$. To determine the absolute plasma levels of T15/EO6 IgM antibodies, we used a capture assay with the T15-specific anti-idiotypic antibody AB1-2 (29). MDA-LDL-immunized mice had significantly higher T15/EO6 plasma levels compared with PBS-immunized mice ( $806 \pm 157$ vs. $277 \pm 68 \mathrm{ng} / \mathrm{ml}, P<0.005$; Figure 4B). To put these increases in perspective, we compared in the same assay the plasma titers of T15/EO6 induced by MDA-LDL to those induced in a prior study by immunization with $S$. pneumoniae, a potent PC antigen that induced a robust increase in T15/EO6 sufficient to inhibit the progression of atherosclerosis (12). These data indicate that immunization with MDA-LDL led to T15/EO6 levels that were on average $35 \%$ of those achieved by immunization with pneumococci (Figure 4C).

IgMs form immune complexes in plasma of MDA-LDL-immunized mice. The increased T15/EO6 titers in MDA-LDL-immunized $L D L R^{-1-}$ mice demonstrated the additional engagement of innate immunity and suggested that EO6 could have contributed to the protective effect of this immunization. Therefore, we tested the possibility that there were higher levels of IgM/apoB immune complexes (ICs) in the plasma of MDA-LDL-immunized mice than in the plasma of PBS-immunized $L D L R^{-/-}$mice. Indeed, mice immunized with MDA-LDL had higher plasma levels of IgM/apoB ICs than the PBS group did (Figure 4D, $P<0.01)$. There were only low levels of circulating IgG/apoB ICs, and these did not differ between the two groups $(0.11 \pm 0.02$ vs. $0.10 \pm 0.02$ relative light units [RLU]/RLU for the MDA and PBS groups, respectively).
IL-5 stimulates B-1 cells to secrete natural antibody T15/EO6. The expansion of T15/EO6 IgM in the MDA-LDL-immunized mice was unexpected, given that MDA-LDL does not bind to T15/EO6 antibodies and thus could not have directly ligated the B-cell receptor of PCspecific B-1 cells, which would lead to the secretion of T15/EO6. Although the secretion of B-1 cell-dependent IgM antibodies is known to be $\mathrm{T}$ cell independent, $\mathrm{T}$ cells are thought to provide noncognate help for B-1 cells (30), and IL-5 has been described as an important factor in B-1 cell biology $(31,32)$.

To directly test the hypothesis that IL-5 derived from the MDALDL-specific T-cell responses provided noncognate $\mathrm{T}$ cell help for B-1 cells to secrete T15/EO6, we isolated B-cell subsets from
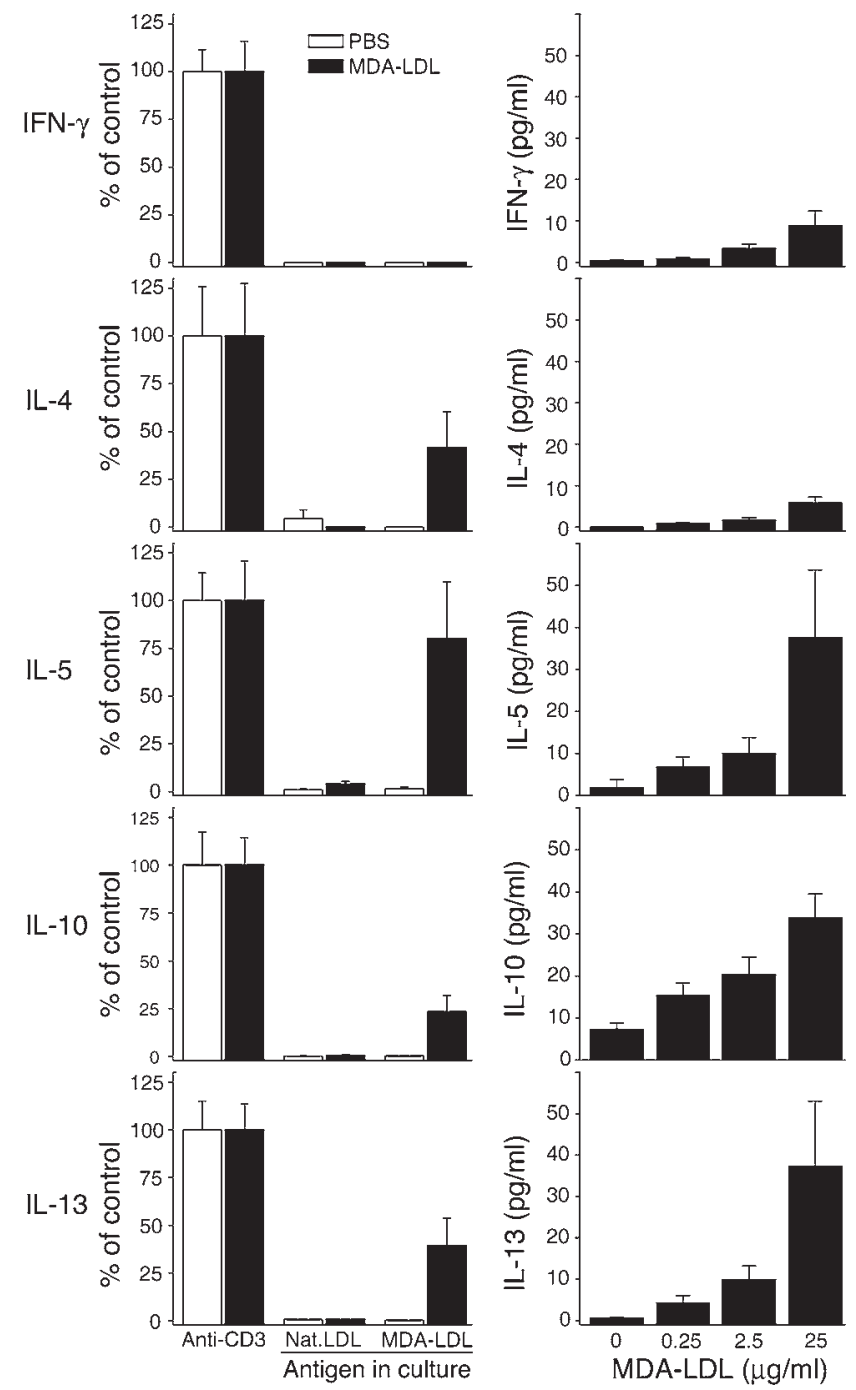

Figure 3

Antigen-specific cytokine secretion of splenocytes from cholesterol-fed $L D L R^{-/-}$mice immunized with MDA-LDL ( $n=10$; black bars) or PBS ( $n=11$; white bars). Left column: Splenocytes were cultured for 72 hours in the presence of anti-CD28 and either anti-CD3, murine native LDL, or murine MDA-LDL, and supernatants were analyzed for cytokines. Data are presented as percentage of the cytokine secretion in parallel cultures maximally stimulated with anti-CD3/CD28 (=100\%). Right column: splenocytes were stimulated either alone or with indicated amounts of murine MDA-LDL (without anti-CD28). Values are mean \pm SEM of splenocyte cultures of all mice from each group. 
A
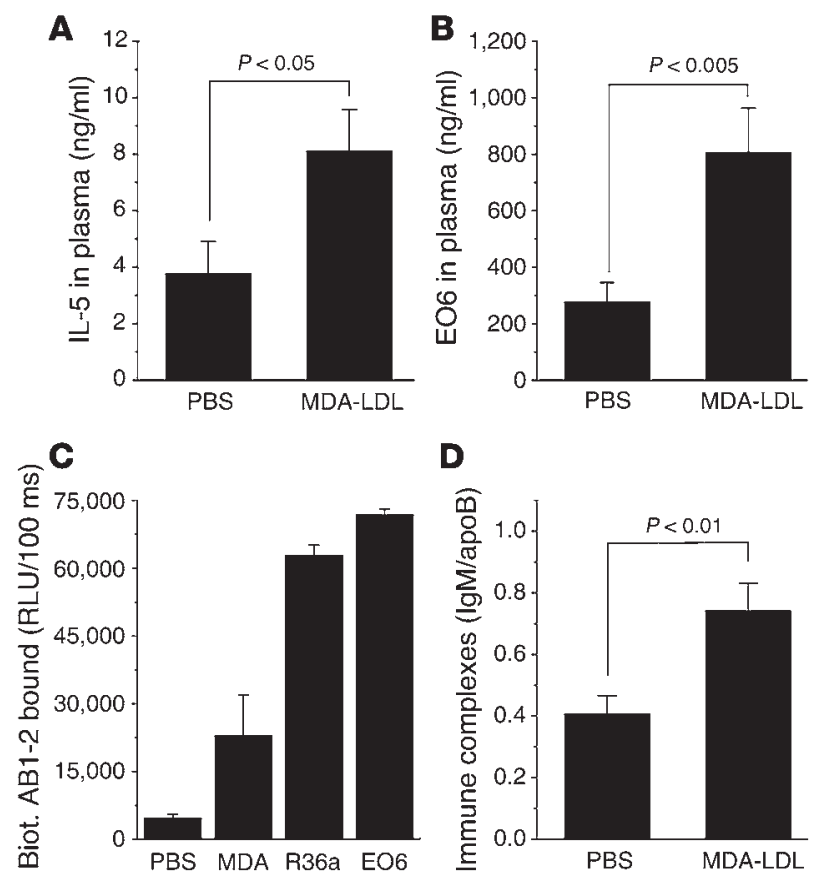

the peritoneal cavity and the spleens of naive C57BL/ 6 mice by FACS, based on the surface expression of CD19 and CD23 (33). Peritoneal B-1 cells were separated from conventional B-2 cells, and splenic B cells were separated into marginal-zone B cells and follicular B cells. These individual subsets of B cells were cultured in growth media alone or with IFN- $\gamma$, IL-4, or IL-5. After 7 days, culture supernatants were harvested and antibody binding to OxLDL was determined by ELISA. Only IL-5 strongly stimulated B-1 cells to secrete IgM antibodies to OxLDL (Figure 5A), and addition of an IL-5 neutralizing antibody abolished this response. None of the other B cell subsets showed equivalent anti-OxLDL IgM secretion following IL-5 stimulation. There was no comparable IgG binding of these culture supernatants (data not shown), which is consistent with the fact that B-1 cells predominantly secrete IgM. In addition, using the anti-idiotypic antibody AB1-2, we definitively demonstrated that the B-1 cells secreted T15/EO6 following IL-5 stimulation (Figure 5B).

We next tested the capacity of IL-5 to induce T15/EO6 antibodies in vivo by daily intraperitoneal injections of naive $\mathrm{C} 57 \mathrm{BL} / 6$ mice with murine recombinant IL-5 for 7 days. On day 8 , there was more than a four-fold increase in plasma T15/EO6 titers in mice injected with IL-5 $(P<0.05)$, compared to vehicle (BSA; Figure 5C).

We analyzed plasma levels of T15/EO6 antibodies in $I L-5^{-/-}$mice, which have been reported to have transiently decreased numbers of B- 1 cells at a young age ( $<8$ weeks). Surprisingly, the baseline levels of T15/EO6 antibodies were strongly affected by the IL-5 deficiency even in adult mice. While 16 weeks old, naive $I L-5^{+/+} \mathrm{C} 57 \mathrm{BL} / 6$ mice had measurable plasma levels of T15/EO6 antibodies at dilutions tested; age-matched $I L-5^{-/-}$mice had no detectable T15/EO6 antibodies (Figure 5D). Interestingly, neither basal IgM levels to OxLDL, nor those to MDA-LDL, differed between the two groups (data not shown), suggesting the existence of other OxLDL-specific IgM antibodies that are not derived from B-1 cells.

Next, we examined whether IL-5 is essential for the induction of T15/EO6 as a consequence of MDA-LDL immunization.

\section{Figure 4}

Increased levels of IL-5 and EO6 in the plasma of cholesterol-fed $L D L R^{-1-}$ mice immunized with MDA-LDL. At time of death, plasma was obtained from mice immunized with MDA-LDL $(n=10)$ and PBS $(n=11)$. (A) IL-5 levels in plasma of MDA-LDL immunized mice are increased. (B) EO6 antibody levels in plasma of MDA-LDL-immunized mice are increased. The amount of EO6 present was determined using an ELISA based capture assay with anti-idiotypic AB1-2 as described in Methods and was calculated based on an EO6 standard curve. (C) Comparison of T15/EO6 antibody titers in $L D L R^{-1-}$ mice immunized with MDA-LDL (current study) versus mice immunized with S. pneumoniae (R36a; $n=9$ ) from a previous study (12). EO6 antibody titers were determined at a plasma dilution of 1:500 in the same assay. Purified EO6 was used as a positive control. Biot., biotinylated. (D) Circulating IgM/apoB ICs are increased in MDA-LDL-immunized mice. Results are expressed as IgM/apoB. All bars represent the mean \pm SEM values of all mice from each intervention group.

$I L-5^{-/-}$and $I L-5^{+/+}$mice (both on a C57BL/6 background) were immunized with murine MDA-LDL. After one primary and two booster immunizations, $I L-5^{-/-}$and $I L-5^{+/+}$mice displayed similar IgG1 (Figure 5E) and IgG2a titers (data not shown) to MDA-LDL, demonstrating that IL-5 deficiency did not impair TD responses. In contrast, the development of IgM titers to MDA-LDL was lower in IL-5 $5^{-/-}$mice than in the $I L-5^{+/+}$control group (data not shown). Most striking, however, was the fact that the induction of IgMs to OxLDL (Figure 5F), and specifically the induction of T15/EO6 (Figure 5G), was greatly impaired in $I L-5^{-/-}$mice, despite three injections (Figure $5, \mathrm{E}-\mathrm{G})$, suggesting a pivotal role for IL-5 in the induction of T15/ EO6 as a consequence of immunization with MDA-LDL.

IL-5 deficiency accelerates atherosclerosis. We asked whether IL-5 itself could play a functional role in atherogenesis, even in the absence of exogenous immunizations. To generate $L D L R^{-/-}$mice with deficient production of IL-5, we transplanted irradiated $L D L R^{-/-}$mice with bone marrow from $I L-5^{-/-}$or $I L-5^{+/+}$mice. Four weeks after bone marrow transplantation (BMT), mice were switched to an atherogenic diet for the subsequent 16 weeks to induce lesion formation. At the time of sacrifice, splenocytes of the $L D L R^{-1-}$ mice with $I L-5^{-/-}$bone marrow showed significantly less production of IL-5 following anti-CD3 stimulation compared with those that received $I L-5^{+/+}$bone marrow (Table 1 ). The production of the cytokines IL-4, IL-10, and IFN- $\gamma$, however, did not differ between the two groups (Table 1). Moreover, production of IL-5 by antiCD3-stimulated peripheral blood cells was virtually absent in recipients of $I L-5^{-/-}$bone marrow (Table 1 ). Consequently, plasma IL-5 levels were significantly decreased in these mice (Table 1). Thus, the $I L-5^{-/-} L D L R^{-/-}$bone marrow chimeras had a selective insufficiency, but not absence, of IL- 5 production compared to the control mice that received $I L-5^{+/+}$bone marrow. En face analyses of the extent of atherosclerosis in the entire aorta revealed a significantly increased degree of lesion formation as a result of transplantation of $I L-5^{-/-}$bone marrow, supporting the hypothesized protective role of IL-5 in atherogenesis (Table 1 and Figure $6 \mathrm{~A})$. In addition, atherosclerosis in the aortic origin of recipients of $I L-5^{-/-}$bone marrow was also significantly greater than that in controls, despite the very advanced extent of atherosclerotic lesion formation (Table 1). To determine whether the defective IL-5 secretion resulted in impaired stimulation of B-1 cells to secrete atheroprotective antibodies, we evaluated the titers of T15/ EO6 antibodies in the plasma of these mice. Indeed, at the time of sacrifice, $I L-5^{-/-} L D L R^{-/-}$bone marrow chimeras had significantly 
lower T15/EO6 plasma titers than the $I L-5^{+/+}$controls (Figure 6B). This was not due to an absolute deficiency of B-1 cells, as both recipient groups had similar numbers of peritoneal B-1 cells at the time of death, as determined by FACS (Table 1). Analyses of autoantibody titers to MDA-LDL and OxLDL revealed decreased levels of TI IgG3 titers but not of TD IgG1 or IgG2a titers (data not shown). T15/EO6 IgM antibodies can bind OxLDL and prevent its uptake by macrophages (7). To determine whether the decreased levels of T15/EO6 antibodies also led to an impaired formation of ICs with minimally oxidized LDL in vivo, we measured the levels of circulating LDL ICs that contained T15/EO6 antibodies. Recipients of $I L-5^{-/-}$bone marrow had significantly lower levels of plasma T15/EO6 antibody-apoB ICs during the period of cholesterol feeding (Figure 6C). IgG-apoB ICs were very low and did not differ between the two recipient groups (data not shown).

\section{Discussion}

Here we report four main findings that are relevant to the impact of adaptive and innate immunity in atherogenesis. First, we show that immunization of mice with homologous MDA-LDL results in a strongly biased TD activation of Th2 cells with specificity for the MDA modification; this bias is intrinsic to the immunogen and independent of hypercholesterolemia (34). Consistent with our previous results $(13,14)$, this immunization ameliorates the progression of atherosclerosis. Second, we show that immunization with MDA-LDL surprisingly also results in a significant expansion of innate T15/EO6 clonotypic antibodies that bind POVPC-like OxPLs, which are typically not present in MDA-LDL. These natural antibodies can block the uptake of OxLDL by macrophages. Their near-monoclonal expansion in mice, which was achieved by immunization with a potent $\mathrm{PC}$-containing pneumococcal preparation, significantly reduced atherosclerosis (12). Remarkably, the MDA-

\section{Figure 5}

Role of IL-5 in the production of T15/EO6 natural antibodies. (A and B) IL-5 stimulates antibody secretion in vitro. Peritoneal B-1 cells (black bars) and B-2 cells (white bars), and splenic marginal zone (MZ) B cells (dark gray bars) and follicular B cells (light gray bars) were cultured for 7 days in either medium alone, or with IFN- $\gamma$, IL-4, or IL-5 with and without anti-IL-5 mAb. Polymyxin B was added to all cultures to neutralize contaminating LPS effects. (A) IgM binding to OxLDL in culture supernatants. (B) T15/EO6 antibodies in culture supernatants, measured with the anti-idiotypic antibody AB1-2. Values are mean RLU \pm SEM from duplicate determinations of triplicate cultures. This experiment was repeated three times. (C) IL-5 stimulates the production of T15/ EO6 antibodies in vivo. C57BL/6 mice received daily intraperitoneal injections with recombinant mouse IL-5 $(n=6)$ or vehicle only (BSA; $n=4$ ) for 7 days, and the amount of T15/EO6 antibodies was determined in the plasma. Shown is the fold increase over the baseline levels at a 1:100 plasma dilution. Bars represent mean \pm SEM of triplicate determinations of individual mice. $P<0.05$, Student's unpaired $t$ test; Welch corrected. (D) Naive IL-5 $5^{-/}$mice have decreased T15/EO6 antibody levels. In $/ L-5^{+/+}$(open circles) and $/ L-5^{-/-}$C57BL/6 mice (filled circles), 15-16 weeks of age (both $n=3$ ), T15/EO6 antibody titers were determined. Shown is the binding of T15/EO6 antibodies of individual plasma samples diluted 1:100. Values are the mean RLU of triplicate determinations. (E-G) Impaired induction of T15/EO6 antibodies in $I L-5^{-/-}$mice immunized with MDA-LDL. $I L-5^{+/+}$(open circles) and IL-5-/- (filled circles) C57BL/6 mice were immunized with MDA-LDL and plasma antibody titers were determined. (E) IgG1 binding to MDA-LDL (F), IgM binding to OxLDL, and (G) T15/EO6 antibodies of individual plasmas at 1:250 dilution before and after immunization. Values are the mean RLU of triplicate determinations.
LDL immunization increased titers of T15/EO6 to levels 35\% as high as those induced by pneumococcal immunization. Third, we define the mechanism by which immunization with MDA-LDL led to expansion of T15/EO6 antibodies. Because the expansion of the B-1 cell-derived T15/EO6 is independent of classic cognate T-cell help (30), it was unclear how the TD response to MDA-LDL led to increased T15/EO6 titers. In these studies, we demonstrate that the induced Th 2 cells produced large amounts of IL-5, which in turn provided noncognate stimulation of B-1 cells to secrete the innate T15/EO6 IgM antibodies $(7,12)$. Fourth, using BMT in which $L D L R^{-1-}$ mice were reconstituted with wild-type or $I L-5^{-/-}$ bone marrow, we establish that IL-5 had an overall atheropro-
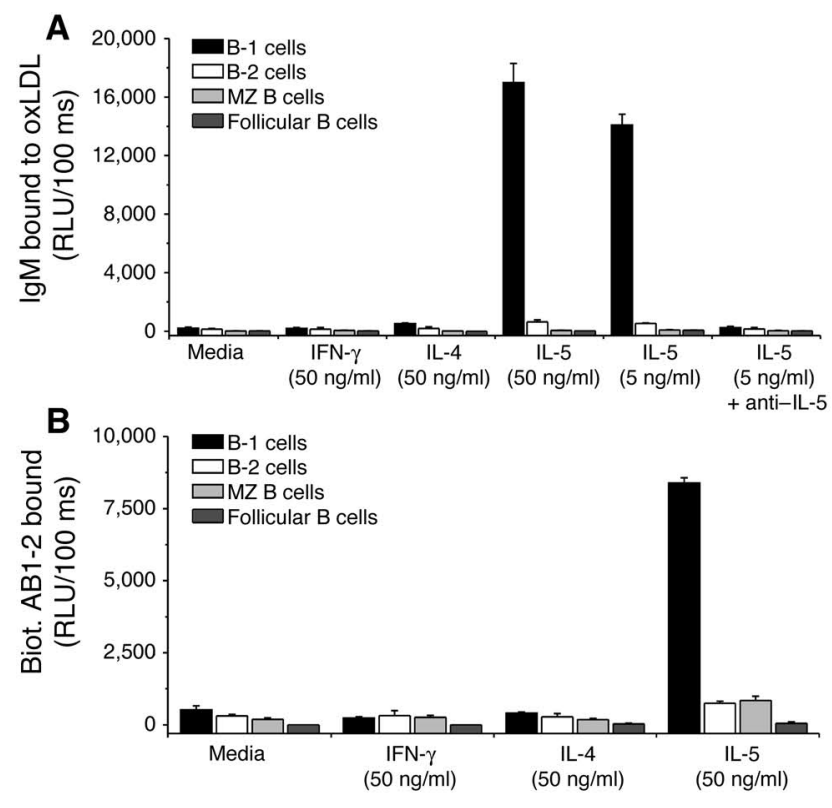

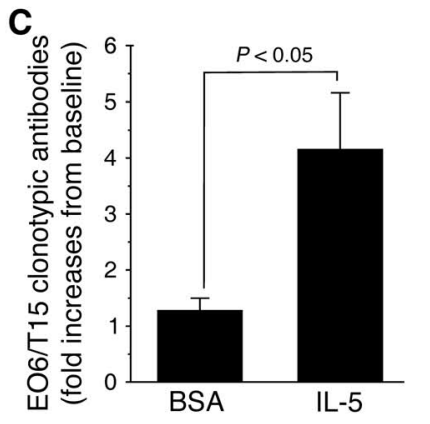

E

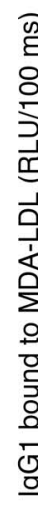

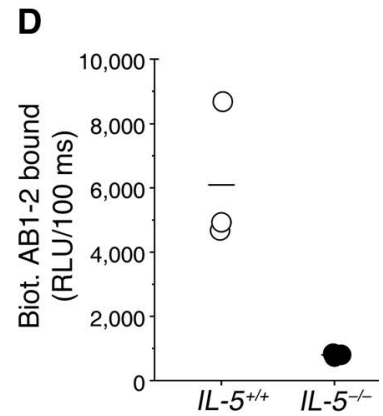

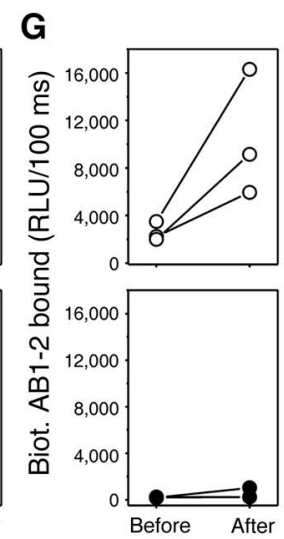


Table 1

Overview of experimental groups from bone marrow transplantation

\begin{tabular}{|c|c|c|}
\hline & $\begin{array}{c}I L-5^{-/-} \rightarrow L D L R^{-/-} \\
\quad(n=15)\end{array}$ & $\begin{array}{c}I L-5^{+/+} \rightarrow L D L R^{-/-} \\
(n=14)\end{array}$ \\
\hline Weights (g) & $22.8 \pm 0.7$ & $23.0 \pm 0.7$ \\
\hline TC (mg/dl) & $776 \pm 39$ & $748 \pm 32$ \\
\hline TG (mg/dl) & $59 \pm 4$ & $65 \pm 5$ \\
\hline \multicolumn{3}{|l|}{ Atherosclerosis } \\
\hline En face (\% of aorta) & $10.5 \pm 1.1^{\mathrm{C}}$ & $6.8 \pm 0.6$ \\
\hline Aortic origin ( $\mathrm{mm}^{2} / \mathrm{sect}$.) & $0.752 \pm 0.026^{D}$ & $0.638 \pm 0.048$ \\
\hline \multicolumn{3}{|l|}{ Cytokines splenocytes ${ }^{A}$} \\
\hline IL-4 (pg/ml) & $32.6 \pm 6.3$ & $42.5 \pm 7.4$ \\
\hline IL-5 (pg/ml) & $36.9 \pm 12.3^{\mathrm{E}}$ & $122.6 \pm 17.7$ \\
\hline IL-10 (pg/ml) & $498.8 \pm 67.8$ & $476.7 \pm 72.6$ \\
\hline IFN $\gamma(\mathrm{ng} / \mathrm{ml})$ & $61.1 \pm 5.7$ & $53.8 \pm 5.9$ \\
\hline IL-5 PBC ${ }^{A}(p g / m l)$ & $1.5 \pm 0.8^{E}$ & $16.4 \pm 2.9$ \\
\hline IL-5 plasma (pg/ml) & $367 \pm 61^{D}$ & $678 \pm 107$ \\
\hline \multicolumn{3}{|l|}{ PEC phenotype ${ }^{B}$} \\
\hline $\operatorname{lgM}+\mathrm{CD}^{+}$(\% of total) & $5.6 \pm 0.6$ & $6.4 \pm 0.7$ \\
\hline IgM+Mac- $1+(\%$ of total $)$ & $22.3 \pm 1.8$ & $22.7 \pm 2.4$ \\
\hline \multicolumn{3}{|c|}{ 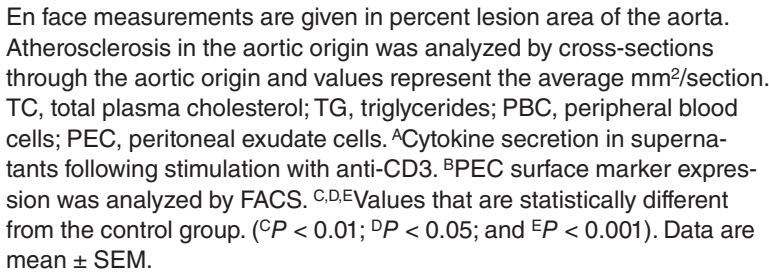 } \\
\hline
\end{tabular}

tective role. Thus, our data demonstrate a mechanism whereby immunization with an oxidation-specific epitope leads to a classic adaptive Th2 response, which in turn leads to the recruitment of natural innate immunity to OxLDL. In aggregate, these events provided atheroprotection. In particular, our data demonstrate that IL-5 links adaptive and natural immunity specific for epitopes of OxLDL and is atheroprotective. These data may explain in part the postulated atheroprotective role of Th2-mediated immunity.

Th1 adaptive responses, and Th1 cytokines in particular have been shown to play a key role in promoting atherogenesis $(17,35-37)$. In contrast, we now demonstrate that immunization with MDA-LDL directly triggers an antigen-specific Th2 response that is associated with an atheroprotective effect. There are a number of possible mechanisms by which such a biased Th 2 response could be atheroprotective in general, as discussed in several recent reviews $(3,17)$. We now specifically demonstrate that MDA-LDL immunization induces a decidedly biased Th2 response characterized by the prominent secretion of IL-5, sufficient to cause increased systemic levels of IL-5 in immunized mice. Moreover, $I L-5^{-/-} L D L R^{-/-}$bone marrow chimeras, which showed decreased production of IL-5, developed significantly more atherosclerosis than recipients of wild-type bone marrow. These mice did not show decreases in their anti-OxLDL IgG1 and IgG2a titers com-

\section{Figure 6}

pared with wild-type mice. Thus, the protective effect of IL-5 seems to be independent of TD IgG responses to OxLDL.

IL-5 has been detected in human $(38,39)$ and murine (our unpublished results) atherosclerotic lesions, although it is irregularly expressed. In our present study, we did not find increased expression of IL-5 mRNA in lesions of MDA-LDL-immunized mice when determined by quantitative PCR analysis (data not shown). Nevertheless, for immunoregulatory functions that affect atherogenesis, IL- 5 need not be expressed in lesions. IL- 5 is mainly produced by Th2 cells and mast cells. As an eosinophil differentiation and maturation factor $(40,41)$, it is widely studied for its role in asthma and allergies, as well as in host defense from intestinal parasites. Interestingly, patients with asthma have been reported to have decreased atherosclerotic disease (42). Eosinophilic infiltrations are rarely found in atherosclerotic lesions (38), and we did not observe differences in peripheral blood eosinophil counts in the recipients of $I L-5^{-/-}$and $I L-5^{+/+}$bone marrow (data not shown).

Importantly, IL-5 also induces proliferation and differentiation of B cells, particularly B-1 cells, which constitutively express the IL-5 receptor (31, 43-45). Accordingly, IL-5 transgenic mice have (in addition to eosinophilia) increased numbers of B-1 cells in the peritoneal cavity and elevated levels of serum $\operatorname{IgM}$ and $\operatorname{IgA}(46)$, while IL-5R $\alpha^{-/-}$mice do not show IL-5-induced enhancement of B cell responses to TI type 2 (TI-2) antigens (47). Immune responses to PC are examples of classic TI-2 responses. In the current studies, we demonstrate that IL-5 stimulated the production of the natural IgM T15/EO6 in vitro and in vivo, whereas mice deficient in IL-5 had virtually no T15/EO6 antibodies in their plasma. Therefore, IL-5 seems to be pivotally involved in the expansion of atheroprotective T15/EO6 natural antibodies derived from B-1 cells $(8,12)$. Indeed, $L D L R^{-/-}$mice that received bone marrow from $I L-5^{-/-}$mice exhibited significantly lower titers of B-1 cell-derived T15/EO6 antibodies and decreased IgG3 titers to OxLDL - another TI-2 response. Consequently, there was also a reduced formation of ICs with minimally oxidized LDL in the plasma of these mice.

Interestingly, we observed a proatherogenic phenotype in the $I L-5^{-/-} L D L R^{-/-}$bone marrow chimeras despite the fact that these

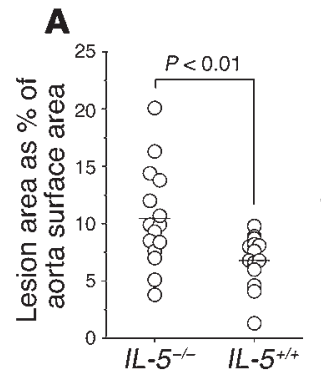

B
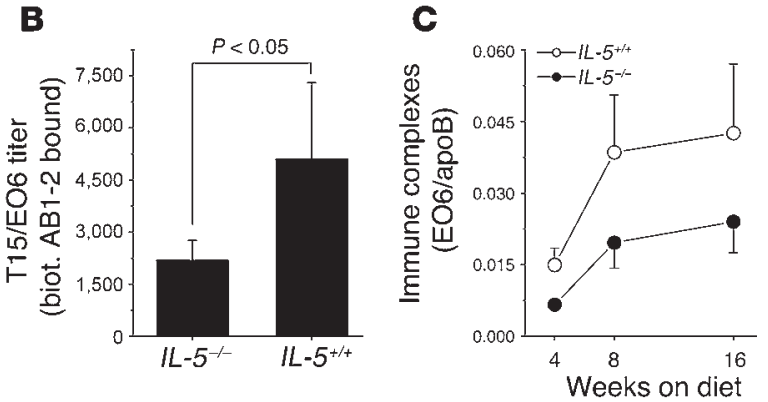

Increased atherosclerosis in IL-5-deficient $L D L R^{-1-}$ mice. $L D L R^{-/-}$mice were reconstituted with bone marrow from either $I L-5^{-/-}$mice $\left(I L-5^{-/-} ; n=15\right)$ or $I L-5^{+/+}$mice $\left(I L-5^{+/+}\right.$; $n=14$ ) and fed an atherogenic diet for 16 weeks. (A) Increased extent of atherosclerosis in aortas of recipients of $I L-5^{-/}$bone marrow $(n=15)$ compared to recipients of $I L-5^{+/+}$ bone marrow $(n=14 ; P<0.01)$. Horizontal bars indicate means of each group. (B) Decreased titers of T15/EO6 antibodies in plasma of $L D L R^{-/-}$mice reconstituted with IL-5 $5^{-/}$bone marrow. Data are mean \pm SEM titers of all mice of each group $(P<0.05)$. (C) Reduced formation of circulating T15/EO6-apoB ICs in recipients of $I L-5^{-/-}$bone marrow. Results are expressed as T15/EO6 antibodies per apoB. Data are the mean \pm SEM values of all mice from each intervention group at 4,8 , and 16 weeks after initiation of the atherogenic diet. $P<0.05$, repeated-measures ANOVA. 
mice still had the capacity to secrete low levels of IL-5. Thus, this BMT model represents an IL-5 hypomorphic phenotype. We are currently cross-breeding $I L-5^{-/-}$mice with $L D L R^{-/-}$mice, which then can be used to assess the full impact of IL- 5 deficiency on atherogenesis, as well as the quantitative role that IL-5 plays in mediating the atheroprotective effect of immunization with MDALDL. Nevertheless, the fact that even reduced IL-5 production led to accelerated lesion formation argues for an important role of IL-5 during atherogenesis. While our results do not unequivocally establish that the atheroprotective effect of IL-5 is mediated by the expansion of atheroprotective T15/EO6 antibodies, our data clearly demonstrate a strong relationship between IL-5 and the secretion of T15/EO6 antibodies.

B-1 cells are the major source of natural IgM antibodies and are prominently involved in TI responses (48). Because multivalent cross-linking of the B cell receptor is not sufficient to drive optimal antibody production, it has been suggested that noncognate interactions with T cells, NK cells and other cells enhance these responses (30). The exact mechanisms underlying such noncognate $T$ cell help are still unknown, but T cells, once activated, could either directly or indirectly costimulate B1 cells. Although many stimuli, including antigen, LPS and cytokines, have been demonstrated to activate B-1 cells, the exact physiological triggers during an orchestrated immune response are not clear. IL-5 specifically plays a role in stimulating IgM secretion by B- 1 cells $(43,49)$. Moreover, IL-10 seems to be involved in B-1 cell activation as well (44, 50 ), although partially in an autocrine manner (51), whereas IL-12 has been shown to inhibit B-1 cell proliferation (52). Thus, it seems that specific Th2 subsets could positively affect B-1 cell activity. Since immunization of wild-type mice with MDA-LDL prominently induced IL-5-secreting Th2 cells and T15/EO6 antibodies, whereas immunization of $I L-5^{-/-}$mice with MDA-LDL failed to induce T15/EO6 antibodies, MDA-specific Th2 cells secreting IL- 5 could represent such a specific Th subset.

Although the stimulation of B-1 cells by IL-5 does not exclusively result in the secretion of T15/EO6, the general concept that B-1 cells have specificity for stress-induced self-structures (53) would allow the recruitment of other natural antibodies that have related specificities, such as to other oxidation-specific epitopes. Consistent with this notion, we found that in vitro incubation of IL-5 with B-1 cells derived from normal mice also stimulated antiMDA-LDL IgM antibodies (unpublished observations), suggesting that MDA epitopes are yet another set of stress-induced self-structures that are also recognized by B-1 cells (54). Importantly, we have previously shown that many monoclonal IgM antibodies derived from the spleens of atherosclerotic apoE $E^{-/-}$mice bind to MDA-LDL (6). Thus, during atherogenesis, a setting in which the generation of oxidation-specific epitopes of OxLDL is greatly increased, oxidation-specific B-1 cells would be preferentially activated through their B cell receptors. In turn, this activation would be helped by the augmented IL-5. Thus, de facto, generalized expansion of B-1 cells in this setting would be expected to preferentially enhance secretion of IgM directed at oxidation-specific epitopes.

As recently demonstrated, without the natural immunoprotection afforded by B cells, atherogenesis can be dramatically accelerated (55). In particular, B-1 cells are primitive tiers of innate immunity and arise spontaneously in mice early in ontogeny (56). T15/EO6 antibodies secreted by B-1 cells are present at low titers even in normal mice but are expanded during atherogenesis, presumably as a result of increased antigenic pressure $(6,8,57)$. These antibod- ies could provide significant protection against atherosclerosis by inhibition of OxLDL uptake by macrophages, removal of proatherogenic particles away from the artery wall, or neutralization of proinflammatory effects of OxPLs. Indeed, mice that have undergone splenectomy, which removes the anatomical site from which B-1 cells secrete antibodies, have accelerated atherogenesis (58).

In conclusion, our findings demonstrate that the adaptive immune response against MDA can in turn efficiently boost the expansion of innate natural T15/EO6 IgM antibodies, which recognize PC, a different oxidation-specific epitope. In this paper we provide direct evidence that this positive modulation of protective natural immunity to OxLDL is mediated by IL-5 and that IL-5 deficiency accelerates atherogenesis. Because strategies are currently being developed to inhibit IL-5 actions in patients with asthma and other allergic diseases $(59,60)$, a detailed understanding of the role of IL-5 in atherogenesis is needed.

\section{Methods}

Immunogens and antigens. Murine LDL, murine MDA-LDL, human MDALDL, and human OxLDL were prepared as described $(7,14)$. All murine preparations used for immunization and cell culture were tested for endotoxin levels by chromogenic Limulus amoebocyte assay (QCL-1000; BioWhittaker Inc, Wakersville, Maryland, USA) and contained less than 1.5 ng lipopolysaccharides/mg protein (ApoB).

Mice, immunizations, and diets. Female C57BL/6 mice, $\mathrm{MHCII}^{--}$and T cell receptor $\beta^{-/-}$mice were from The Jackson Laboratories (Bar Harbor, Maine, USA), and $I L-5^{-/-}$mice were a kind gift of Manfred Kopf (ETH Zürich, Zürich, Switzerland) (32). All mice were on a C57BL/6 background and were bred and maintained in our colony. All mice used were more than 8 weeks of age and fed nonatherogenic rodent chow (TD8604; Harlan Teklad, Madison, Wisconsin, USA). Primary immunizations consisted of $50 \mu \mathrm{g}$ murine MDA-LDL in $100 \mu \mathrm{l}$ sterile PBS emulsified in an equal volume of complete Freund's adjuvant (Sigma-Aldrich, St. Louis, Missouri, USA) and were injected subcutaneously into both inguinal regions. Booster immunizations consisted of $25 \mu \mathrm{g}$ antigen in $100 \mu \mathrm{l}$ sterile PBS emulsified in incomplete Freund's adjuvant (Sigma-Aldrich) and were given intraperitoneally every $2-3$ weeks. If no adjuvant was used, the same amounts of antigen were given in $200 \mu \mathrm{L}$ sterile PBS.

For the atherosclerosis intervention study, 24 female $L D L R^{-1-}$ mice (tenth generation C57BL/6), 11-13 weeks of age, were divided into equal groups matched for body weight, age, and plasma cholesterol. Mice in the MDA group $(n=12)$ were immunized with murine MDA-LDL in Freund's adjuvant, and mice in the PBS group $(n=12)$ received PBS in Freund's adjuvant. Mice were initially fed nonatherogenic chow and received the primary and one booster immunization on day 14 . On day 17 , mice were switched to an atherogenic diet containing $21.2 \%$ milk fat and $1.25 \%$ cholesterol (TD96121; Harlan Teklad) for the 13 remaining weeks during which they received three more booster immunizations every 3-4 weeks. Three mice (one in the PBS group and two in the MDA group) were a priori excluded from the final analysis because they developed skin lesions, loss of weight and/or dropping plasma cholesterol levels.

BMT studies were performed as previously described (61). Thirty 8-weekold female $L D L R^{-/-}$mice on a C57BL/6 background, bred at the Scripps Research Institute Animal Facility, were given 10-Gy lethal total body-irradiation to eliminate endogenous bone marrow stem cells and bone marrow-derived cells. Bone marrow cells used for repopulation were extracted from the femur and tibia of three female $I L-5^{-/}$and three female $I L-5^{+/+}$mice (8 weeks old, C57BL/ 6 background). Irradiated mice were injected intravenously with $2 \times 10^{6}$ bone marrow cells harvested from either $I L-5^{-/-}(n=15)$ or $I L-5^{+/+}$mice $(n=15)$. Mice were fed regular chow for 4 weeks after BMT to 
allow for bone marrow reconstitution and then switched to an atherogenic diet containing $15.8 \%$ fat and $1.25 \%$ cholesterol (TD94059; Harlan Teklad) for an additional 16 weeks to induce lesion formation. One mouse from the $I L-5^{+/+}$control group was excluded from the formal analysis because plasma cholesterol levels were more than 2.5 standard deviations above the mean of all mice. Differences in the extent of atherosclerosis between groups would remain significant even if this mouse were included. All experimental protocols were approved by the Animal Subjects Committee at University of California, San Diego, and by the Institutional Animal Care and Use Committee (IACUC) of The Scripps Research Institute.

Plasma cholesterol and triglyceride levels were determined using automated enzymatic assays (Roche Diagnostics, Indianapolis, Indiana, USA; Equal Diagnostics, Exton, Pennsylvania, USA) and are presented as time averages, that is, the area under the cholesterol curve over time divided by days of cholesterol feeding. Plasma lipoprotein profiles were determined by FPLC as described previously (62).

Immunoassays. Specific antibody titers were determined by chemiluminescent enzyme immunoassays as previously described (7). To determine the plasma titers of specific antibody binding to given antigens, plasmas were serially diluted and antibody binding measured by chemiluminescent ELISA. A titer was defined as the reciprocal of the maximal dilution at which binding of the secondary antibody was two times above the background binding. Several secondary antibodies were used: alkaline phosphatase-labeled (AP-labeled) goat anti-mouse IgM ( $\mu$-chain specific) and IgG ( $\gamma$-chain specific) (Sigma-Aldrich); AP-labeled rat anti-mouse IgG1 (LO-MG1-2) (Zymed Laboratories Inc., South San Francisco, California, USA); and AP-labeled rat anti-mouse IgG2a (R19-15) and rat anti-mouse IgG3 (clone R40-82) (BD Biosciences Pharmingen, San Diego, California, USA).

Flow cytometry. At time of death, peritoneal exudate cells (PEC) were harvested by peritoneal lavage using ice-cold PBS supplemented with $1 \%$ BSA. After blocking with a specific anti-Fc receptor monoclonal antibody (clone 2.4G2, BD Biosciences - Pharmingen) for 10 minutes at $4^{\circ} \mathrm{C}, 10^{6}$ cells were stained with the fluorescently labeled monoclonal antibodies (FITC-labeled anti-IgM [II/41], phycoerythrin-labeled [PE-labeled] anti-CD5 [53-7.3], and APC-labeled anti-CD11b/Mac-1 [M1/70]; all BD Biosciences - Pharmingen) in $100 \mu \mathrm{L}$ volumes of staining buffer for 30 minutes at $4{ }^{\circ} \mathrm{C}$ in darkness, followed by two washes of large volumes of staining buffer. Cell populations were analyzed by multiparameter flow cytometry using a BD FACSCalibur or a FACScan instrument (BD, San Jose, California, USA). More than $10^{5}$ cells were analyzed per sample, with dead cells excluded by forward and side scatter properties. Surface marker analysis was performed using CellQuest software (BD).

Splenocyte proliferation assay. Splenocytes were suspended in culture media (RPMI 1640 media containing 10\% heat-inactivated fetal calf serum, 10 $\mathrm{mM}$ Hepes buffer, $2 \mathrm{mM}$ L-glutamine, $0.1 \mathrm{mM}$ nonessential amino acid solution, $100 \mathrm{U} / \mathrm{ml}$ penicillin, $100 \mu \mathrm{g} / \mathrm{ml}$ streptomycin and $0.05 \mathrm{mM}$ 2-mercaptoethanol; all Invitrogen, Carlsbad, California, USA) and seeded at $2 \times 10^{5}$ cells per well in 96-well flat-bottom plates in final culture volumes of $200 \mu \mathrm{l}$. Triplicate cultures of splenocytes from individual mice were incubated in the absence or presence of titrated amounts of antigen for 72 hours at $37^{\circ} \mathrm{C} / 5 \% \mathrm{CO}_{2}$ and then pulsed with $1 \mu \mathrm{Ci}\left[{ }^{3} \mathrm{H}\right]$-thymidine (ICN Biomedicals, Irvine, California, USA) per well for 18 hours, after which cells were harvested onto filter paper using a cell harvester (Wallac; PerkinElmer, Gaithersburg, Maryland, USA). Thymidine incorporation was determined by liquid scintillation counting. Values are presented as cellular $\left[{ }^{3} \mathrm{H}\right]$-thymidine uptake in cpm per well from which values for wells with media alone were subtracted.

Enzyme-linked immunospot (ELISpot) assay for single-cell IFN- $\gamma$ and IL-5 secretion. Splenocytes from individual mice were cultured in $100 \mu \mathrm{l}$ culture media with and without $25 \mu \mathrm{g} / \mathrm{ml}$ of murine MDA-LDL in duplicate at $2 \times 10^{6}$ and $1 \times 10^{6}$ cells/well in washed and blocked 96-well MultiScreen-HA sterile nitrocellulose plates (Millipore, Bedford, Massachusetts, USA), which had been coated overnight with capture antibodies for IFN- $\gamma$ (R4-6A2, BD Biosciences - Pharmingen) or IL-5 (TRFK5, BD Biosciences - Pharmingen). In some assays, parallel incubations were performed in the presence of 10 $\mu \mathrm{g} / \mathrm{ml}$ anti-CD28 (37.51, BD Biosciences - Pharmingen). After 22 hours incubation at $37^{\circ} \mathrm{C} / 5 \% \mathrm{CO}_{2}$ cells were removed by washing and antigenspecific cytokine secreting spot-forming cells (SFC) were detected using matched biotinylated antibodies against IFN- $\gamma$ (XMG1.2, BD Biosciences - Pharmingen) or IL-5 (TRFK4, BD Biosciences - Pharmingen), followed by HRP-Streptavidin (Zymed Inc.). Plates were developed using a tetramethylbenzidine membrane substrate system (Kirkegaard \& Perry Laboratories, Gaithersburg, Maryland, USA), and spots were quantified by examination either under a dissecting microscope or using an automated ImmunoSpot Image Analyzer (Cellular Technology, Cleveland, Ohio, USA).

Cytokine release assay and ELISA. Splenocytes $\left(5 \times 10^{5}\right)$ were seeded into 96-well round-bottom plates in culture media in a final volume of $200 \mu \mathrm{l}$. Triplicate cultures of splenocytes from individual mice were incubated for 72 hours at $37^{\circ} \mathrm{C} / 5 \% \mathrm{CO}_{2}$ in culture media with and without titrated amounts of antigen (unless indicated otherwise, results represent incubations with $25 \mu \mathrm{g} / \mathrm{ml}$ antigen). In some assays, parallel incubations were performed in the presence of $10 \mu \mathrm{g} / \mathrm{ml}$ anti-CD28. In separate wells, cells were cultured in the presence of $10 \mu \mathrm{g} / \mathrm{ml}$ plate-bound anti-CD3 (145-2C11, BD Biosciences - Pharmingen) and $10 \mu \mathrm{g} / \mathrm{ml}$ soluble anti-CD28 (37.51, BD Biosciences - Pharmingen). For the stimulation of peripheral blood lymphocytes, blood was obtained in heparinized tubes, and after red blood cell lysis, $2.5 \times 10^{5}$ nucleated cells were cultured for 48 hours with and without plate bound anti-CD3. The amounts of specific cytokines in supernatants were determined by a chemiluminescent-based sandwich ELISA using matched pairs of specific antibodies for capture and detection, followed by incubation steps with AP-labeled NeutrAvidin (Pierce Biotechnology Inc., Rockford, Illinois, USA) and LumiPhos 530 (Lumigen Inc., Southfield, Michigan, USA). The following antibodies were paired with appropriate biotinylated detecting antibodies (R\&D Systems, Minneapolis, Minnesota, USA): anti-IFN- $\gamma$ 37801.11/37875.11, anti-IL-4 30340.11, anti-IL-5 TRFK5, anti-IL-10 JES052A5, and anti-IL-13 38213.11. The recombinant cytokines used for standardization were purchased from R\&D Systems. The detection limit for all cytokines was approximately $10 \mathrm{pg} / \mathrm{ml}$, and plasma was used at dilutions of $1: 5$ to $1: 25$.

Evaluation of atherosclerosis. The extent of atherosclerosis was determined in a blinded fashion in en face preparations of the arch or the entire aorta, as well as in cross sections through the aortic origin, by computer-assisted image analysis as previously described $(61,63)$.

Measurement of T15 clonotypic antibodies. For the detection of T15 clonotypic antibodies (e.g., EO6) in the plasma of mice, a chemiluminescentbased double antibody capture assay was developed and validated, using the monoclonal anti-T15-idiotypic antibody AB1-2, a mouse IgG1, which is absolutely specific for both the canonical $\mathrm{T} 15 \mathrm{~V}_{\mathrm{H}}$ and the T15 $\mathrm{V}_{\mathrm{L}}$ regions (29) (a kind gift of J.F. Kearney, University of Alabama at Birmingham, Birmingham, Alabama). For the assay, $5 \mu \mathrm{g} / \mathrm{ml}$ of AB1-2 or an IgG1 isotype control MOPC31c (Sigma-Aldrich) in PBS were coated onto microtitration plates overnight at $4{ }^{\circ} \mathrm{C}$. After washing and blocking steps, $50 \mu \mathrm{l}$ of murine plasma diluted stepwise or at a given dilution in BSAPBS were incubated overnight at $4^{\circ} \mathrm{C}$. After further washing, captured T15-clonotypic antibodies were detected using $0.1 \mu \mathrm{g} / \mathrm{ml}$ biotinylated AB1-2 diluted in BSA-PBS, followed by AP-labeled NeutrAvidin (Pierce Biotechnology Inc.) diluted in BSA-TBS, and a 50\% aqueous solution of LumiPhos 530. Biotinylated antibodies were prepared using the EZLink Biotin Hydrazide method (Pierce Biotechnology Inc.) according to 
the manufacturer's protocol. Purified EO6 and EO14, which is an IgM monoclonal autoantibody specific for MDA-LDL and not T15 clonotypic (8), were used as positive and negative controls, respectively (6). In some experiments a standard curve was constructed with EO6.

ApoB-100 IC measurement. ApoB-100 particles were captured on microtiter wells using LF3, a monoclonal antibody specific for murine apoB-100 (64) (kindly provided by S.G. Young, Gladstone Institute of Cardiovascular Disease, San Francisco, California, USA), which was coated on microtiter wells at $5 \mu \mathrm{g} / \mathrm{ml}$ in PBS. After washing and blocking steps, plasmas (1:100 in BSA-PBS) were incubated in wells for 1 hour at room temperature and after further washing, bound IgM or IgG was detected using AP-conjugated goat anti-mouse IgM or anti-IgG by chemiluminescent ELISA. For detection of T15/EO6 antibodies bound to the captured apoB-containing particles, biotinylated AB1-2 was used, followed by incubation with AP-labeled NeutrAvidin and LumiPhos 530. In parallel wells, the relative amount of apoB captured in each sample was determined using biotinylated LF5, another monoclonal antibody specific for mouse apoB-100 (64), followed by incubation with AP-labeled NeutrAvidin and LumiPhos 530. Because LF5 binds to only one epitope of apoB-100, the amount of each antibody used in this assay bound to the captured LDL was then normalized for the amount of captured apoB, and expressed as a ratio of antibody counts (RLU/100ms) to apoB-100 counts (RLU/100ms).

$B$-cell isolation and stimulation assay. Splenocytes and peritoneal exudate cells were isolated from adult naive $\mathrm{C} 57 \mathrm{BL} / 6$ mice. After red blood cell lysis and a 2 -hour incubation at $37^{\circ} \mathrm{C} / 5 \% \mathrm{CO}_{2}$ in culture medium, nonadherent cells were incubated with an anti-Fc $\gamma \mathrm{R}$ antibody (2.4G2, BD Biosciences - Pharmingen), followed by staining with PE-labeled anti-CD19 (1D3, BD Biosciences - Pharmingen) and FITC-labeled anti-CD23 (B3B4, BD Biosciences - Pharmingen). Washed cells were sorted at the Flow Cytometry Core Facility at UCSD using a FACS Vantage SE (BD, San Jose, California, USA). Post-sort analysis revealed a purity of greater than $93 \%$ for B-1 cells $\left(\mathrm{CD} 19^{+} / \mathrm{CD} 23^{-}\right)$, greater than $94 \%$ for B-2 cells $\left(\mathrm{CD} 19^{+} / \mathrm{CD} 23^{+}\right)$, greater than $74 \%$ for marginal-zone (MZ) B cells (CD19+/CD23-), and greater than $99 \%$ for follicular B cells $\left(\mathrm{CD} 19^{+} / \mathrm{CD} 23^{+}\right)$. For stimulation assays of sort-purified B cells, $4 \times 10^{4}$ cells were incubated in 96-well flat-bottom plates with indicated concentrations of recombinant mouse cytokines (BD Biosciences - Pharmingen) or an equivalent volume of vehicle (BSA) in triplicate cultures in culture media in final culture volumes of $200 \mu \mathrm{l}$ for 7 days at $37^{\circ} \mathrm{C} / 5 \% \mathrm{CO}_{2}$. Culture supernatants were harvested, diluted 1:1 in BSAPBS, and antibody binding was determined by ELISA. In indicated cultures, cells were also incubated in the presence of $0.5 \mu \mathrm{g} / \mathrm{ml}$ anti-IL-5 (TRFK5, $\mathrm{BD}$ Biosciences - Pharmingen). Because B cells are particularly sensitive to endotoxins (65) and low amounts of endotoxin were measured in the BSA used, parallel assays were performed in the presence of $0.1 \mathrm{mg} / \mathrm{ml}$ Polymyxin B (Sigma-Aldrich) to neutralize potential endotoxin activity. This concentration was effective but not toxic to either of the cell types tested, as determined in pilot experiments.

IL-5 injections. Sixteen-week-old female C57BL/6 mice received daily intraperitoneal injections of $200 \mathrm{ng}$ recombinant mouse IL-5 (BD Biosciences - Pharmingen) or equal volumes of vehicle (BSA) alone in $200 \mu \mathrm{l}$ PBS for 7 days. Plasma was obtained at baseline and one day after the last injection.

Statistical analysis. Data are presented as mean \pm SEM. Results were analyzed by one-way ANOVA and Student's unpaired $t$ test, unless indicated differently. $P<0.05$ was considered significant.

\section{Acknowledgments}

We thank Gregg Silverman for helpful discussions. This work was supported by NIH grants HL56989 (SCOR in Molecular Medicine and Atherosclerosis), HL69464 and HL57505 (to J.L. Witztum), and HL35297 and HL43815 (to L.K. Curtiss). C.J. Binder was supported by a Ph.D. scholarship from the Boehringer Ingelheim Fonds, a scholarship from the Austrian Academy of Science, and a fellowship from the American Heart Association (AHA, Western Affiliates). K. Hartvigsen was supported by grants from the NovoNordisk/Danish-American Foundation, the Reinholdt W. Jorck \& Hustrus Foundation, the Otto Monsteds Foundation, the Arvid Nilssons Foundation, the Villum Kann Rasmussen Foundation, and a fellowship from the AHA (Western Affiliates). M.-K. Chang was supported by a scientist development grant from the AHA. We are grateful to Manfred Kopf for provision of the IL-5/- mice. We thank Gary Bradshaw, Joshua Bulgrien, Susan Butler, Florencia Casanada, Brian Crain, Joseph Juliano, Elizabeth Miller, Jennifer Pattison, and Mercedes Silvestre for excellent technical assistance; and Dennis J. Young in the UCSD Cancer Center Flow Cytometry Shared Resource for flow cytometer expertise.

Received for publication November 6, 2003, and accepted in revised form May 25, 2004.

Address correspondence to: Christoph J. Binder, or Joseph L. Witztum, Department of Medicine, 0682, University of California, San Diego, 9500 Gilman Drive, La Jolla, California 92093-0682, USA. Phone: (858) 534-4402; Fax: (858) 534-2005; E-mail: cbinder@ucsd. edu (C.J.B.), jwitztum@ucsd.edu (J.L.W.).
1. Steinberg, D. 2002. Atherogenesis in perspective: hypercholesterolemia and inflammation as partners in crime. Nat. Med. 8:1211-1217.

2. Glass, C.K., and Witztum, J.L. 2001. Atherosclerosis: the road ahead. Cell. 104:503-516.

3. Binder, C.J., et al. 2002. Innate and acquired immunity in atherogenesis. Nat. Med. 8:1218-1226.

4. Hansson, G.K., Libby, P., Schonbeck, U., and Yan, Z.Q. 2002. Innate and adaptive immunity in the pathogenesis of atherosclerosis. Circ. Res. 91:281-291.

5. Hörkkö, S., et al. 2000. Immunological responses to oxidized LDL. Free Radic. Biol. Med. 28:1771-1779.

6. Palinski, W., et al. 1996. Cloning of monoclonal autoantibodies to epitopes of oxidized lipoproteins from apolipoprotein E-deficient mice. Demonstration of epitopes of oxidized low density lipoprotein in human plasma. J. Clin. Invest. 98:800-814.

7. Hörkkö, S., et al. 1999. Monoclonal autoantibodies specific for oxidized phospholipids or oxidized phospholipid-protein adducts inhibit macrophage uptake of oxidized low-density lipoproteins. J. Clin.
Invest. 103:117-128.

8. Shaw, P.X., et al. 2000. Natural antibodies with the T15 idiotype may act in atherosclerosis, apoptotic clearance, and protective immunity. J. Clin. Invest. 105:1731-1740.

9. Friedman, P., Hörkkö, S., Steinberg, D., Witztum, J.L., and Dennis, E.A. 2002. Correlation of antiphospholipid antibody recognition with the structure of synthetic oxidized phospholipids. Importance of Schiff base formation and aldol concentration. J. Biol. Chem. 277:7010-7020.

10. Briles, D.E., Forman, C., Hudak, S., and Claflin, J.L. 1982. Anti-phosphorylcholine antibodies of the T15 idiotype are optimally protective against Streptococcus pneumoniae. J. Exp. Med. 156:1177-1185.

11. Harnett, W., and Harnett, M.M. 1999. Phosphorylcholine: friend or foe of the immune system? Immunol. Today. 20:125-129.

12. Binder, C.J., et al. 2003. Pneumococcal vaccination decreases atherosclerotic lesion formation: Molecular mimicry between Streptococcus pneumoniae and oxidized LDL. Nat. Med. 9:736-743.
13. Palinski, W., Miller, E., and Witztum, J.L. 1995. Immunization of low density lipoprotein (LDL) receptordeficient rabbits with homologous malondialdehyde-modified LDL reduces atherogenesis. Proc. Natl. Acad. Sci. U. S. A. 92:821-825.

14. Freigang, S., Hörkkö, S., Miller, E., Witztum, J.L., and Palinski, W. 1998. Immunization of LDL receptor-deficient mice with homologous malondialdehyde-modified and native LDL reduces progression of atherosclerosis by mechanisms other than induction of high titers of antibodies to oxidative neoepitopes. Arterioscler. Thromb. Vasc. Biol. 18:1972-1982.

15. George, J., et al. 1998. Hyperimmunization of apo-Edeficient mice with homologous malondialdehyde low-density lipoprotein suppresses early atherogenesis. Atherosclerosis. 138:147-152.

16. Zhou, X., Caligiuri, G., Hamsten, A., Lefvert, A.K., and Hansson, G.K. 2001. LDL immunization induces T-cell-dependent antibody formation and protection against atherosclerosis. Arterioscler. Thromb. Vasc. Biol. 21:108-114. 
17. Hansson, G.K. 2001. Immune mechanisms in atherosclerosis. Arterioscler. Thromb. Vasc. Biol. 21:1876-1890

18. Palinski, W., et al. 1989. Low density lipoprotein undergoes oxidative modification in vivo. Proc. Natl. Acad. Sci. U. S. A. 86:1372-1376.

19. Palinski, W., Tangirala, R.K., Miller, E., Young, S.G., and Witztum, J.L. 1995. Increased autoantibody titers against epitopes of oxidized LDL in LDL receptor-deficient mice with increased atherosclerosis. Arterioscler. Thromb. Vasc. Biol. 15:1569-1576.

20. Palinski, W., et al. 1994. ApoE-deficient mice are a model of lipoprotein oxidation in atherogenesis. Demonstration of oxidation-specific epitopes in lesions and high titers of autoantibodies to malondialdehyde-lysine in serum. Arterioscler. Thromb. 14:605-616.

21. Zhou, X., Paulsson, G., Stemme, S., and Hansson, G.K. 1998. Hypercholesterolemia is associated with a T helper (Th) $1 /$ Th2 switch of the autoimmune response in atherosclerotic apo E-knockout mice. J. Clin. Invest. 101:1717-1725.

22. Lee, T.S., Yen, H.C., Pan, C.C., and Chau, L.Y. 1999. The role of interleukin 12 in the development of atherosclerosis in ApoE-deficient mice. Arterioscler. Thromb. Vasc. Biol. 19:734-742.

23. Huber, S.A., Sakkinen, P., David, C., Newell, M.K., and Tracy, R.P. 2001. T helper-cell phenotype regulates atherosclerosis in mice under conditions of mild hypercholesterolemia. Circulation. 103:2610-2616.

24. King, V.L., Szilvassy, S.J., and Daugherty, A. 2002. Interleukin-4 deficiency decreases atherosclerotic lesion formation in a site-specific manner in female LDL receptor-/- mice. Arterioscler. Thromb. Vasc. Biol. 22:456-461.

25. Davenport, P., and Tipping, P.G. 2003. The role of interleukin-4 and interleukin-12 in the progression of atherosclerosis in apolipoprotein E-deficient mice. Am. J. Pathol. 163:1117-1125.

26. Mallat, Z., et al. 1999. Protective role of interleukin10 in atherosclerosis. Circ. Res. 85:E17-E24.

27. Pinderski, L.J., et al. 2002. Overexpression of interleukin- 10 by activated $\mathrm{T}$ lymphocytes inhibits atherosclerosis in LDL receptor-deficient mice by altering lymphocyte and macrophage phenotypes. Circ. Res. 90:1064-1071.

28. Billiau, A., and Matthys, P. 2001. Modes of action of Freund's adjuvants in experimental models of autoimmune diseases. J. Lenkoc. Biol. 70:849-860.

29. Kearney, J.F., Barletta, R., Quan, Z.S., and Quintans, J. 1981. Monoclonal vs. heterogeneous anti-H-8 antibodies in the analysis of the antiphosphorylcholine response in BALB/c mice. Eur. J. Immunol. 11:877-883.

30. Snapper, C.M., et al. 2001. Distinct types of T-cell help for the induction of a humoral immune response to Streptococcus pneumoniae. Trends Immunol. 22:308-311.

31. Takatsu, K. 1998. Interleukin 5 and B cell differentiation. Cytokine Growth Factor Rev. 9:25-35.

32. Kopf, M., et al. 1996. IL-5-deficient mice have a developmental defect in CD5+ B-1 cells and lack eosinophilia but have normal antibody and cytotoxic T cell responses. Immunity. 4:15-24.

33. Erickson, L.D., Foy, T.M., and Waldschmidt, T.J. 2001.
Murine B1 B cells require IL-5 for optimal T celldependent activation. J. Immunol. 166:1531-1539.

34. Robertson, A.K., Zhou, X., Strandvik, B., and Hansson, G.K. 2004. Severe hypercholesterolaemia leads to strong Th2 responses to an exogenous antigen. Scand. J. Immunol. 59:285-293.

35. Gupta, S., et al. 1997. IFN-gamma potentiates atherosclerosis in ApoE knock-out mice. J. Clin. Invest. 99:2752-2761.

36. Whitman, S.C., Ravisankar, P., and Daugherty, A. 2002. IFN-gamma deficiency exerts gender-specific effects on atherogenesis in apolipoprotein $\mathrm{E}^{-/-}$mice. J. Interferon Cytokine Res. 22:661-670.

37. Song, L., Leung, C., and Schindler, C. 2001. Lymphocytes are important in early atherosclerosis. J. Clin. Invest. 108:251-259. doi:10.1172/ JCI200111380.

38. Frostegard, J., et al. 1999. Cytokine expression in advanced human atherosclerotic plaques: dominance of pro-inflammatory (Th1) and macrophagestimulating cytokines. Atherosclerosis. 145:33-43.

39. Schönbeck, U., Sukhova, G.K., Gerdes, N., and Libby, P. 2002. $\mathrm{T}(\mathrm{H}) 2$ predominant immune responses prevail in human abdominal aortic aneurysm. Am. J. Pathol. 161:499-506.

40. Yamaguchi, Y., et al. 1988. Highly purified murine interleukin 5 (IL-5) stimulates eosinophil function and prolongs in vitro survival. IL-5 as an eosinophil chemotactic factor. J. Exp. Med. 167:1737-1742.

41. Yamaguchi, Y., et al. 1988. Purified interleukin 5 supports the terminal differentiation and proliferation of murine eosinophilic precursors. J. Exp. Med. 167:43-56.

42. Lasser, E.C., Berry, C., and Kortman, K. 1987. Diminished atherosclerotic arterial calcifications in asthma. A possible role for elevated endogenous heparin-like material. Allergy. 42:549-552.

43. Wetzel, G.D. 1989. Interleukin 5 regulation of peritoneal Ly-1 B lymphocyte proliferation, differentiation and autoantibody secretion. Eur. J. Immunol. 19:1701-1707.

44. Nisitani, S., Tsubata, T., Murakami, M., and Honjo, T. 1995. Administration of interleukin-5 or -10 activates peritoneal B-1 cells and induces autoimmune hemolytic anemia in anti-erythrocyte autoantibodytransgenic mice. Eur. J. Immunol. 25:3047-3052.

45. Sakiyama, T., Ikuta, K., Nisitani, S., Takatsu, K., and Honjo, T. 1999. Requirement of IL-5 for induction of autoimmune hemolytic anemia in anti-red blood cell autoantibody transgenic mice. Int. Immunol. 11:995-1000.

46. Tominaga, A., et al. 1991. Transgenic mice expressing a B cell growth and differentiation factor gene (interleukin 5) develop eosinophilia and autoantibody production. J. Exp. Med. 173:429-437.

47. Yoshida, T., et al. 1996. Defective B-1 cell development and impaired immunity against Angiostrongylus cantonensis in IL-5R alpha-deficient mice. Immunity. 4:483-494.

48. Berland, R., and Wortis, H.H. 2002. Origins and functions of B-1 cells with notes on the role of CD5. Annu. Rev. Immunol. 20:253-300.

49. Tominaga, A., et al. 1989. Establishment of IL-5 dependent early B cell lines by long-term bone marrow cultures. Growth Factors. 1:135-146.
50. Ishida, H., Hastings, R., Kearney, J., and Howard, M. 1992. Continuous anti-interleukin 10 antibody administration depletes mice of Ly-1 B cells but not conventional B cells. J. Exp. Med. 175:1213-1220.

51. O'Garra, A., et al. 1992. Ly-1 B (B-1) cells are the main source of B cell-derived interleukin 10. Eur. J. Immunol. 22:711-717.

52. Vogel, L.A., Lester, T.L., Van Cleave, V.H., and Metzger, D.W. 1996. Inhibition of murine B1 lymphocytes by interleukin-12. Eur. J. Immunol. 26:219-223.

53. Bendelac, A., Bonneville, M., and Kearney, J.F. 2001. Autoreactivity by design: innate B and T lymphocytes. Nat. Rev. Immunol. 1:177-186.

54. Chang, M.K., et al. 1999. Monoclonal antibodies against oxidized low-density lipoprotein bind to apoptotic cells and inhibit their phagocytosis by elicited macrophages: evidence that oxidation-specific epitopes mediate macrophage recognition. Proc. Natl. Acad. Sci. U. S. A. 96:6353-6358.

55. Major, A.S., Fazio, S., and Linton, M.F. 2002. B-lymphocyte deficiency increases atherosclerosis in LDL receptor-null mice. Arterioscler. Thromb. Vasc. Biol. 22:1892-1898.

56. Stall, A.M., Wells, S.M., and Lam, K.P. 1996. B-1 cells: unique origins and functions. Semin. Immunol. 8:45-59.

57. Kearney, J.F. 2000. Immune recognition of OxLDL in atherosclerosis [comment]. J. Clin. Invest. 105:1683-1685.

58. Caligiuri, G., Nicoletti, A., Poirier, B., and Hansson, G.K. 2002. Protective immunity against atherosclerosis carried by B cells of hypercholesterolemic mice. J. Clin. Invest. 109:745-753. doi:10.1172/ JCI200207272.

59. Leckie, M.J. 2003. Anti-interleukin-5 monoclonal antibodies: preclinical and clinical evidence in asthma models. Am. J. Respir. Med. 2:245-259.

60. Danzig, M., and Cuss, F. 1997. Inhibition of interleukin-5 with a monoclonal antibody attenuates allergic inflammation. Allergy. 52:787-794.

61. Schiller, N.K., Kubo, N., Boisvert, W.A., and Curtiss, L.K. 2001. Effect of gamma-irradiation and bone marrow transplantation on atherosclerosis in LDL receptor-deficient mice. Arterioscler. Thromb. Vasc. Biol. 21:1674-1680.

62. Li, A.C., et al. 2000. Peroxisome proliferator-activated receptor gamma ligands inhibit development of atherosclerosis in LDL receptor-deficient mice. J. Clin. Invest. 106:523-531.

63. Tangirala, R.K., Rubin, E.M., and Palinski, W. 1995. Quantitation of atherosclerosis in murine models: correlation between lesions in the aortic origin and in the entire aorta, and differences in the extent of lesions between sexes in LDL receptor-deficient and apolipoprotein E-deficient mice. J. Lipid. Res. 36:2320-2328.

64. Zlot, C.H., et al. 1999. Generation of monoclonal antibodies specific for mouse apolipoprotein B100 in apolipoprotein B-48-only mice. J. Lipid. Res. 40:76-84.

65. Wetzel, G.D. 1991. Induction of interleukin-5 responsiveness in resting B cells by engagement of the antigen receptor and perception of a second polyclonal activation signal. Cell Immunol. 137:358-366. 